\title{
AN INTEGRATED PROBABILISTIC MODEL FOR ASSESSING A NANOCOMPONENT'S RELIABILITY
}

\author{
NADER EBRAHIMI,* Northern Illinois University \\ YARONG YANG, ${ }^{* *}$ University of California, Berkeley
}

\begin{abstract}
We construct an integrated probabilistic model to capture interactions between atoms of a nanocomponent. We then use this model to assess reliabilities of nanocomponents with different structures. Several properties of our proposed model are also described under a sparseness condition. The model is an extension of our previous model based on Markovian random field theory. The proposed integrated model is flexible in that pairwise relationship information among atoms as well as features of individual atoms can be easily incorporated. An important feature that distinguishes the integrated probabilistic model from our previous model is that the integrated approach uses all available sources of information with different weights for different types of interaction. In this paper we consider the nanocomponent at a fixed moment of time, say the present moment, and we assume that the present state of the nanocomponent depends only on the present states of its atoms.
\end{abstract}

Keywords: Associated random variable; conditionally increasing in sequence; Gibbs distribution; Gibbs sampling; Markov random field; reliability

2010 Mathematics Subject Classification: Primary 60N05

Secondary 60G55; 90B25

\section{Introduction}

Over the past several years, nanoscience and nanotechnology have become two of the most important fields at the forefront of physics, chemistry, engineering, mathematical science, and biology. Nanoscience lies at the intersection of traditional science and engineering, quantum mechanics, and the most basic processes of life itself. Nanotechnology, on the other hand, encompasses how we harness our knowledge of nanoscience to fabricate nanocomponents and nanosystems. The Greek word 'nano' meaning dwarf, refers to a reduction of size or time by $10^{-9}$, which is one thousand times smaller than a micron. Equivalent to ten angstroms, one nanometer $(\mathrm{nm})$ is one billionth of a meter.

In both fields, much attention has been given to the dual problem of designing nanocomponents with novel physical properties and how they can be fabricated. Receiving less attention has been the question of the nanocomponent reliability. Reliability measures the ability of a nanocomponent to perform its intended function. Today, high reliability is necessary to guarantee the advancement and utilization of nanocomponents due to the fact that they account for a high proportion of costs of newly designed nanosystems and multiscale systems.

Received 17 February 2011; revision received 29 May 2011.

* Postal address: Division of Statistics, Northern Illinois University, DeKalb, IL 60115, USA.

Email address: nader@math.niu.edu

** Postal address: Department of Civil and Environmental Engineering, University of California, Berkeley, CA 94720, USA. 
Ebrahimi (2008), (2010) developed a Markov random field (MRF) model for capturing atomatom interaction. He then used this model to assess reliabilities of one-dimensional and twodimensional nanocomponents. One major problem with this model is that it ignores different types of interaction that might exist between the atoms as well as features of individual atoms. Note that we refer to a 'nanocomponent' as the component which is made of atoms.

In this paper we extend the MRF-based method to create an integrated approach that can easily incorporate different types of interaction. We then use the proposed model to assess the reliability of a nanocomponent. Throughout the paper, the reliability of a nanocomponent is defined as the probability of surviving up to a known mission time or the present moment, say $t_{0}$.

The paper is organized as follows. In Section 2 we describe the integrated MRF model. In Section 3 we study several properties of the model under a sparseness condition. In Section 4 we apply the model to predict the limiting reliability of a nanocomponent using a variety of different information as well as different structures for a nanocomponent under our sparseness condition. We obtain bounds for reliabilities of nanocomponents under different structures in Section 4. In this section we also provide the Gibbs sampler method to approximate the reliability of a nanocomponent under different structures and implement it using the R package. Finally, concluding remarks are given in Section 5.

\section{Integrated MRF model}

Consider a nanocomponent consisting of $N$ atoms $A=\{1,2, \ldots, N\}$. Assume that there are available a set of spatial locations $\left\{s_{i}, i=1, \ldots, N\right\}$, where $s_{i}=\left(u_{i}, v_{i}, w_{i}\right)$ denotes the location of atom $i$ in space.

To indicate the state of each atom, define the nonnegative continuous random variable $T\left(s_{i}\right)$, where $T\left(s_{i}\right)$ represents the time that the atom at location $s_{i}, i=1, \ldots, N$, is displaced. Usually, displacement of an atom occurs by switching or breaking a bond. There are many reasons for breaking bonds between atoms. For example, microwave energy can break bonds. Generally speaking, atoms form bonds to become more stable and be able to release energy. However, breaking bonds requires energy. In this paper, the survival of the atom located at $s_{i}$ is defined as the survival up to a known mission time or the present moment $t_{0}, i=1, \ldots, N$.

We capture this by defining the binary random variables in terms of $T\left(s_{1}\right), \ldots, T\left(s_{N}\right)$ and $t_{0}$ by

$$
X\left(s_{i}\right)=\left\{\begin{array}{ll}
1, & T\left(s_{i}\right) \leq t_{0}, \\
0, & T\left(s_{i}\right)>t_{0},
\end{array} \quad i=1, \ldots, N .\right.
$$

In the following $X\left(s_{i}\right)$ will be the random variable and $x\left(s_{i}\right)$ will be its observed value. Let $p_{i}$ be the probability that the atom $i$ is displaced before $t_{0}$, i.e. $p_{i}=\mathrm{P}\left(X\left(s_{i}\right)=1\right)=\mathrm{P}\left(T\left(s_{i}\right) \leq t_{0}\right)$, $i=1, \ldots, N$. Here, for simplicity, we assume that $p_{1}=p_{2}=\cdots=p_{N}=p$. The strength of the bonds define $p$. If $p$ is small then bonds are strong. If $p$ is large then bonds are weak.

Without considering the interaction between atoms,

$$
\mathrm{P}\left(X\left(s_{i}\right)=x\left(s_{i}\right), i=1, \ldots, N\right)=\left(\frac{p}{1-p}\right)^{x}(1-p)^{N},
$$

where $x=\sum_{i=1}^{N} x\left(s_{i}\right)$.

Suppose now that there are interactions among atoms. For a fixed site $s$, define a neighborhood $N^{*}(s)$. Also, define a clique $c$ as any set of sites such that if $s_{i}, s_{j} \in c$ then $s_{j}$ is in the neighborhood of $s_{i}, s_{j} \in N^{*}\left(s_{i}\right)$, and, of course, $s_{i}$ is in the neighborhood of 
$s_{j}, s_{i} \in N^{*}\left(s_{j}\right)$. The MRF model provides a probabilistic framework for obtaining the joint distribution of $X\left(s_{1}\right), \ldots, X\left(s_{N}\right), p\left(x\left(s_{1}\right), \ldots, x\left(s_{n}\right)\right)=\mathrm{P}\left(X\left(s_{i}\right)=x\left(s_{i}\right), i=1, \ldots, N\right)$, via a neighborhood system. Here the state of any random variable, say $X\left(s_{i}\right)$, is assumed to be independent of all other random variables given those of immediate neighbors. More formally,

$$
\mathrm{P}\left(X\left(s_{i}\right)=x_{i} \mid X\left(s_{j}\right)=x_{j} \text { for } i \neq j\right)=\mathrm{P}\left(X\left(s_{i}\right)=x_{i} \mid X(s)=x_{s} \text { for } s \in N^{*}\left(s_{i}\right)\right) .
$$

The Hammersley-Clifford theorem asserts that the joint distribution $p\left(x\left(s_{1}\right), \ldots, x\left(s_{n}\right)\right)$ is the Gibbs distribution and factorizes over the cliques. That is,

$$
p\left(x\left(s_{1}\right), \ldots, x\left(s_{N}\right)\right)=\frac{1}{Z} \exp \left\{-\sum_{c \in C} H_{c}\left(x_{c}\right)\right\},
$$

where $Z$ is the normalizing constant, $C$ is the set of all cliques, $H_{c}$ is the potential function associated with clique $c$, and $x_{c}$ is the assignment of states to the members of $c$. For more details, we refer the reader to Besag (1974). Since it is common to assign 0 potentials to all cliques of size greater than 2 , (1) reduces to

$$
p\left(x\left(s_{1}\right), \ldots, x\left(s_{N}\right)\right)=\frac{1}{Z} \exp \left\{-\sum_{i=1}^{N} H_{1}\left(x\left(s_{i}\right)\right)-\sum_{i, j} H_{2}\left(x\left(s_{i}\right), x\left(s_{j}\right)\right)\right\} .
$$

For our setup, let $E$ be the set of all pairwise interactions among atoms. Clearly, there are three classes of interactions in $E: 1 \leftrightarrow 1$ interactions where both atoms are displaced, $1 \leftrightarrow 0$ interactions where only one of the two interacting atoms has been displaced, and $0 \leftrightarrow 0$ interactions where neither atom is displaced. Let $M_{1}, M_{2}$, and $M_{3}$ be the total number of pairwise interactions for the above classes in $E$. One natural model for $\sum_{(i, j) \in E} H_{2}\left(x\left(s_{i}\right), x\left(s_{j}\right)\right)$ is $-\beta_{1} M_{1}-\beta_{2} M_{2}-\beta_{3} M_{3}$, where $\beta_{1}, \beta_{2}$, and $\beta_{3}$ are the weights of the three classes of interactions. It is clear that

$$
\begin{aligned}
\sum_{(i, j) \in E} H_{2}\left(x\left(s_{i}\right), x\left(s_{j}\right)\right) & =-\beta_{1} M_{1}-\beta_{2} M_{2}-\beta_{3}\left[|E|-M_{1}-M_{2}\right] \\
& =\left(\beta_{3}-\beta_{1}\right) M_{1}+\left(\beta_{3}-\beta_{2}\right) M_{2}-\beta_{3}|E|,
\end{aligned}
$$

where $|E|$ is the number of elements in $E$. Thus, (2) reduces to

$$
p\left(x\left(s_{1}\right), \ldots,\left(s_{N}\right)\right)=\frac{1}{Z_{1}} \exp \left\{\alpha \sum_{i=1}^{N} x\left(s_{i}\right)+\left(\beta_{1}-\beta_{3}\right) M_{1}+\left(\beta_{2}-\beta_{3}\right) M_{2}\right\}
$$

where $\alpha=\log (p / q), Z_{1}$ is a normalizing constant, and $q=1-p$. A modified version of (3) was considered in Deng et al. (2004) for the functional prediction of proteins.

Note that (3) can be written as

$$
\begin{aligned}
p\left(x\left(s_{1}\right), \ldots, x\left(s_{n}\right)\right)= & \left(M\left(\left(\beta_{1}-\beta_{3}\right),\left(\beta_{2}-\beta_{3}\right)\right)\right)^{-1} p^{x} q^{N-x} \\
& \times \exp \left(\left(\beta_{1}-\beta_{3}\right) M_{1}+\left(\beta_{2}-\beta_{3}\right) M_{2}\right),
\end{aligned}
$$

where $x=\sum_{i=1}^{N} x\left(s_{i}\right)$ and $M\left(\left(\beta_{1}-\beta_{3}\right),\left(\beta_{2}-\beta_{3}\right)\right)$ is the joint moment generating function of $Y_{1}(N)=\sum_{(i, j) \in E} X\left(s_{i}\right) X\left(s_{j}\right)$ and $Y_{2}(N)=\left(\sum_{(i, j) \in E} X\left(s_{i}\right)\left(1-X\left(s_{j}\right)\right)+\sum_{(i, j) \in E} X\left(s_{j}\right) \times\right.$ $\left(1-X\left(s_{i}\right)\right)$ ) evaluated at $\beta_{1}-\beta_{3}$ and $\beta_{2}-\beta_{3}$, and computed under the assumption that the 
$X\left(s_{i}\right)$ s are independent and identically distributed Bernoulli variables. It should be noted that in (4) if $\beta_{1}-\beta_{3}=0$ and $\beta_{2}-\beta_{3}=0$, which implies that $\beta_{1}=\beta_{2}=\beta_{3}=0$, then we get independence. Now, based on the general model (4), we have

$$
\begin{aligned}
\mathrm{P}\left(X\left(s_{i}\right)\right. & \left.=1 \mid X\left(s_{j}\right), j=1, \ldots, N \text { and } j \neq i\right) \\
& =\frac{\exp \left\{\alpha+\left(\beta_{1}-\beta_{2}\right) L_{1}^{(i)}+\left(\beta_{2}-\beta_{3}\right) L_{0}^{(i)}\right\}}{1+\exp \left\{\alpha+\left(\beta_{1}-\beta_{2}\right) L_{1}^{(i)}+\left(\beta_{2}-\beta_{3}\right) L_{0}^{(i)}\right\}} \\
& =\frac{p \exp \left\{\left(\beta_{1}-\beta_{2}\right) L_{1}^{(i)}+\left(\beta_{2}-\beta_{3}\right) L_{0}^{(i)}\right\}}{p \exp \left\{\left(\beta_{1}-\beta_{2}\right) L_{1}^{(i)}+\left(\beta_{2}-\beta_{3}\right) L_{0}^{(i)}\right\}+q},
\end{aligned}
$$

where $L_{1}^{(i)}$ is the number of neighbors of atom $i$ that are displaced and $L_{0}^{(i)}$ is the number of neighbors of atom $i$ that are not displaced. That is,

$$
L_{1}^{(i)}=\#\left\{j \in N^{*}\left(s_{i}\right): X\left(s_{j}\right)=1\right\} \quad \text { and } \quad L_{0}^{(i)}=\#\left\{j \in N^{*}\left(s_{i}\right): X\left(s_{j}\right)=0\right\} .
$$

Suppose that the weights of the three classes are such that $\beta_{1} \geq \beta_{2}$ and $\beta_{3} \geq \beta_{2}$. Then, from (5), it is clear that if the number of neighboring atoms that are in state 1 increases, i.e. $L_{1}^{(i)}$ increases, then $L_{0}^{(i)}$ decreases, and, thus, the chance that atom $i$ is in state 1 increases.

The following result provides useful bounds on the joint survival function and the joint cumulative distribution function of $X\left(s_{1}\right), \ldots, X\left(s_{N}\right)$.

Lemma 1. If $\beta_{1} \geq \beta_{2}$ and $\beta_{3} \geq \beta_{2}$, then

(a) $\mathrm{P}\left(X\left(s_{i}\right) \leq x\left(s_{i}\right), i=1, \ldots, N\right) \geq \prod_{i=1}^{N} \mathrm{P}\left(X\left(s_{i}\right) \leq x\left(s_{i}\right)\right)$,

(b) $\mathrm{P}\left(X\left(s_{i}\right) \geq x\left(s_{i}\right), i=1, \ldots, N\right) \geq \prod_{i=1}^{N} \mathrm{P}\left(X\left(s_{i}\right) \geq x\left(s_{i}\right)\right)$.

Proof. From (5), it is clear that $X\left(s_{1}\right), \ldots, X\left(s_{N}\right)$ are conditionally increasing in sequence. It follows immediately from Theorem 4.7 of Barlow and Proschan (1981) that they are associated and, hence, the results follow.

\section{Limiting results under the sparseness condition}

For many nanocomponents, the number of atoms $N$ is very large and it is very unlikely that an atom will be displaced, i.e. $p$ approaches 0 . In this section we give limiting results for our proposed model (4) when $N \rightarrow \infty$ and the random variables $X\left(s_{i}\right)$ which equal 1 are, in a sense made clear below, sparse.

The sparseness condition which we impose is

$$
N p^{2} \rightarrow \lambda
$$

for some $\lambda>0$ when $N \rightarrow \infty$. A similar condition was used in Ebrahimi (2008) to establish his limiting results. Intuitively speaking, condition (6) simply says that $N p^{2}$ remains fixed at $\lambda>0$ or $N p^{2}$ approaches $\lambda>0$ if the number of atoms is large and the bonds between atoms are very strong.

From (6), it is clear that $p \approx \sqrt{\lambda} / \sqrt{N}$ and, thus,

$$
N p(1-p) \approx N \frac{\sqrt{\lambda}}{\sqrt{N}}\left(1-\frac{\sqrt{\lambda}}{\sqrt{N}}\right)=\sqrt{N \lambda}-\lambda \rightarrow \infty \quad \text { as } N \rightarrow \infty .
$$


Now we have the following theorem. It should be noted that the sparseness condition provided in (6) is a sufficient condition for obtaining Theorem 1 below and subsequently the limiting reliability in Section 4.1. There might be other sparseness conditions different from (6), where Theorem 1 is still valid.

Theorem 1. Suppose that $\beta_{1}=\beta_{2}=\beta_{3}=0, p \rightarrow 0, N \rightarrow \infty$, and that condition (6) holds. Then,

(a) $Y_{1}(N)=\sum_{(i, j) \in E} X\left(s_{i}\right) X\left(s_{j}\right) \stackrel{L}{\rightarrow} Y_{1}$,

(b) $Y_{2}(N)=\sum_{(i, j) \in E}\left[X\left(s_{i}\right)\left(1-X\left(s_{j}\right)\right)+X\left(s_{j}\right)\left(1-X\left(s_{i}\right)\right)\right] \stackrel{L}{\rightarrow} Y_{2}$,

where $Y_{1}$ is a Poisson random variable with expected value $\lambda a,\left(Y_{2}-\mathrm{E}\left(Y_{2}\right)\right) / \sqrt{\operatorname{var}\left(Y_{2}\right)}$ is the standard normal, and $Y_{1}$ and $Y_{2}$ are independent. Here $a=\#\left\{j \in N^{*}\left(s_{i}\right)\right\}, i=1, \ldots, N$, $\mathrm{E}\left(Y_{2}\right)=2 a N p q, \operatorname{var}\left(Y_{2}\right)=a N p q[(1-4 p q)(a+N)+4 p q]$, and ' $\stackrel{L}{\rightarrow}$ 'denotes convergence in distribution.

Proof. If $\beta_{1}=\beta_{2}=\beta_{3}=0$ then the variables $X\left(s_{i}\right)$ are independent and identically distributed Bernoulli random variables having success parameter $p$. Now part (a) follows by using Theorem 1 of Saunders et al. (1979). To prove part (b), it is clear that

$$
\begin{aligned}
& \mathrm{E}\left(X\left(s_{i}\right)\left(1-X\left(s_{j}\right)\right)+X\left(s_{j}\right)\left(1-X\left(s_{i}\right)\right)\right)=2 p q, \\
& \operatorname{var}\left(X\left(s_{i}\right)\left(1-X\left(s_{j}\right)\right)+X\left(s_{j}\right)\left(1-X\left(s_{i}\right)\right)\right)=2 p q-4 p^{2} q^{2}, \\
& \operatorname{cov}\left(X\left(s_{i}\right)\left(1-X\left(s_{j}\right)\right)+X\left(s_{j}\right)\left(1-X\left(s_{i}\right)\right), X\left(s_{i}\right)\left(1-X\left(s_{j^{\prime}}\right)\right)+X\left(s_{j^{\prime}}\right)\left(1-X\left(s_{i}\right)\right)\right) \\
& \quad=p q^{2}+p^{2} q-4 p^{2} q^{2} \\
& \quad=p q-4 p^{2} q^{2}, \quad j, j^{\prime} \in N^{*}\left(s_{i}\right),
\end{aligned}
$$

and

$$
\begin{aligned}
& \operatorname{cov}\left(X\left(s_{i}\right)\left(1-X\left(s_{j}\right)\right)+X\left(s_{j}\right)\left(1-X\left(s_{i}\right)\right), X\left(s_{i^{\prime}}\right)\left(1-X\left(s_{j}\right)\right)+X\left(s_{j}\right)\left(1-X\left(s_{i^{\prime}}\right)\right)\right) \\
& \quad=p q-4 p^{2} q^{2}, \quad j \in N^{*}\left(s_{i}\right) \text { and } N^{*}\left(s_{i^{\prime}}\right) .
\end{aligned}
$$

It follows that

$$
\mathrm{E}\left(Y_{2}(N)\right)=2 a N p q
$$

and

$$
\operatorname{var}\left(Y_{2}(N)\right)=a N p q[(1-4 p q)(a+N)+4 p q] .
$$

The result follows from Bloemena (1964), and (7) and (8).

Also, the result $Y_{1}$ and $Y_{2}$ are independent comes from the fact that

$$
\begin{aligned}
\mathrm{P}(X & \left.\left(s_{i}\right) X\left(s_{j}\right)=0, X\left(s_{i}\right)\left(1-X\left(s_{j}\right)\right)+X\left(s_{j}\right)\left(1-X\left(s_{i}\right)\right)=0\right) \\
& =\mathrm{P}\left(X\left(s_{i}\right)=0\right) \mathrm{P}\left(X\left(s_{j}\right)=0\right) \\
& =q^{2} \\
& \approx\left(1-p^{2}\right)(1-2 p q) \\
& =\mathrm{P}\left(X\left(s_{i}\right) X\left(s_{j}\right)=0\right) \mathrm{P}\left(X\left(s_{i}\right)\left(1-X\left(s_{j}\right)\right)+X\left(s_{j}\right)\left(1-X\left(s_{i}\right)\right)=0\right) .
\end{aligned}
$$

This completes the proof. 
Using Theorem 1 , we can replace $M\left(\left(\beta_{1}-\beta_{3}\right),\left(\beta_{2}-\beta_{3}\right)\right)$ in (4) by

$$
\begin{aligned}
M\left(\left(\beta_{1}-\beta_{3}\right),\left(\beta_{2}-\beta_{3}\right)\right) \approx & \exp \left\{-\lambda a+\lambda a \exp \left\{\beta_{1}-\beta_{3}\right\}\right\} \\
& \times \exp \left\{\left(\beta_{2}-\beta_{3}\right) \mathrm{E}\left(Y_{2}\right)+\frac{1}{2}\left(\beta_{2}-\beta_{3}\right)^{2} \operatorname{var}\left(Y_{2}\right)\right\}
\end{aligned}
$$

if condition (6) holds. In (9), $\mathrm{E}\left(Y_{2}\right)$ and $\operatorname{var}\left(Y_{2}\right)$ are given by (7) and (8), respectively.

We note that, for our proposed model (4), when the sparseness condition holds,

$$
\begin{aligned}
\mathrm{P}(X(i) & \left.=1 \mid X\left(s_{j}\right), j=1, \ldots, N, j \neq i\right) \\
& \approx \frac{\sqrt{\lambda} \exp \left\{\left(\beta_{1}-\beta_{2}\right) L_{1}^{(i)}+\left(\beta_{2}-\beta_{3}\right) L_{0}^{(i)}\right\}}{\sqrt{\lambda} \exp \left\{\left(\beta_{1}-\beta_{2}\right) L_{1}^{(i)}+\left(\beta_{2}-\beta_{3}\right) L_{0}^{(i)}\right\}+\sqrt{N}-\sqrt{\lambda}} \\
& =0 .
\end{aligned}
$$

\section{Assessing the reliability of a nanocomponent}

In this section we focus on two types of nanocomponent. Both describe the relationship between the reliability of a nanocomponent and its atoms at $t_{0}$. Recall that the reliability is defined as the probability of survival up to the known time $t_{0}$.

Type I. We assume that the nanocomponent survives if none of the atoms are displaced.

Type II. We assume that the nanocomponent survives if at least $k$ atoms out of $N$ atoms are not displaced or at least one of the neighbors to each of these $k$ atoms is not displaced. For the special case that $k=N$, the nanocomponent survives if any atom or some of its neighbors are not displaced. For $k=1$, the nanocomponent survives if there exists at least one atom such that either this atom or some of its neighbors are not displaced.

It should be noted that both Type I and Type II nanocomponents can be one-dimensional, two-dimensional, or three-dimensional. For definitions of one-dimensional, two-dimensional, and three-dimensional nanocomponents, see Ebrahimi (2010).

As an example, consider a molecular bridge. Suppose that there are $N$ atoms holding the bridge. Clearly, if certain numbers of these atoms disassociate from the bridge then the bridge will fail.

For the Type I nanocomponent, under our proposed model (4), the reliability is

$$
\begin{aligned}
R_{1}\left(N, p, \beta_{1}, \beta_{2}, \beta_{3}\right) & =\mathrm{P}\left(T\left(s_{i}\right)>t_{0}, i=1, \ldots, N\right) \\
& =\mathrm{P}\left(X\left(s_{i}\right)=0, i=1, \ldots, N\right) \\
& =q^{N} M^{-1}\left(\beta_{1}-\beta_{3}, \beta_{2}-\beta_{3}\right) .
\end{aligned}
$$

For the Type II nanocomponent, let $V_{i}$ be the event that the atom located at $s_{i}$ and its neighbor are both displaced by time $t_{0}$, and let $V_{i}^{\prime}$ be the complement of $V_{i}, i=1, \ldots, N$. Also, let $\Omega(k, N)$ be the set of locations $s_{i_{1}}, \ldots, s_{i_{k}}$ such that $1 \leq i_{1}<i_{2}<\cdots<i_{k} \leq N$. It is clear that the reliability is

$$
R_{2}\left(N, p, k, \beta_{1}, \beta_{2}, \beta_{3}\right)=\mathrm{P}\left(\bigcup_{\Omega(k, N)}\left(V_{i_{1}}^{\prime} \cap V_{i_{2}}^{\prime} \cap \cdots \cap V_{i_{k}}^{\prime}\right)\right) .
$$

Note that we do not need to include the remaining $N-k$ in (11) since they are represented by terms of the form $\cdots \cap\left(V_{i_{k+1}} \cup V_{i_{k+1}}^{\prime}\right) \cap \cdots$. 
If we define $Y_{i}^{*}=X\left(s_{i}\right) \prod_{j \in N^{*}\left(s_{i}\right)} X\left(s_{j}\right), i=1, \ldots, N$ and $W_{N}=N-\sum_{i=1}^{N} Y_{i}^{*}$. Then, we can easily show that (11) is equivalent to

$$
R_{2}\left(N, p, k, \beta_{1}, \beta_{2}, \beta_{3}\right)=\mathrm{P}\left(W_{N} \geq k\right)=\mathrm{P}\left(\sum_{i=1}^{N} Y_{i}^{*} \leq N-k\right) .
$$

\subsection{Assessing limiting reliabilities of Type I and Type II nanocomponents under the sparseness condition}

In this section we provide limiting reliabilities when the number of atoms is very large $(N \rightarrow \infty)$ and displacement of an atom is very rare.

The following result gives reliabilities of Type I and Type II nanocomponents under the sparseness condition.

Theorem 2. If $N \rightarrow \infty, p \rightarrow 0$, and $N p^{2} \rightarrow \lambda$, then

(a) $R_{1}\left(N, p, \beta_{1}, \beta_{2}, \beta_{3}\right)=q^{N}\left[\exp \left\{-\lambda a+\lambda a \exp \left\{\beta_{1}-\beta_{3}\right\}\right\}\right.$

$$
\left.\times\left[\exp \left\{\beta_{2}-\beta_{3}\right\} \mathrm{E}\left(Y_{2}\right)+\frac{1}{2}\left(\beta_{2}-\beta_{3}\right)^{2} \operatorname{var}\left(Y_{2}\right)\right]\right]^{-1},
$$

(b) $R_{2}\left(N, p, k, \beta_{1}, \beta_{2}, \beta_{3}\right)=1$.

Here $\mathrm{E}\left(Y_{2}\right)$ and $\operatorname{var}\left(Y_{2}\right)$ are given by $(7)$ and $(8)$, respectively.

Proof. Part (a) follows from Theorem 1. For part (b), first take $k=N$ and assume sparseness:

$$
\begin{aligned}
R_{2}\left(N, p, N, \beta_{1}, \beta_{2}, \beta_{3}\right)= & \mathrm{P}\left(\max _{1 \leq i \leq N} X\left(s_{i}\right) \prod_{j \in N^{*}\left(s_{i}\right)} X\left(s_{j}\right)=0\right) \\
\geq & \mathrm{P}\left(X\left(s_{i}\right) \prod_{j \in N^{*}\left(s_{i}\right)} X\left(s_{j}\right)=0\right) \\
= & 1-\mathrm{P}\left(X\left(s_{i}\right) \prod_{j \in N^{*}\left(s_{i}\right)} X\left(s_{j}\right)=1\right) \\
= & 1-\mathrm{P}\left(X\left(s_{i}\right)=1 \mid X\left(s_{j}\right)=1, j \in N^{*}\left(s_{i}\right)\right) \\
= & \quad \times \mathrm{P}\left(X\left(s_{j}\right)=1, j \in N^{*}\left(s_{i}\right)\right)
\end{aligned}
$$

The last equality comes from (10).

The last equality above implies that $R_{2}\left(N, p, N, \beta_{1}, \beta_{2}, \beta_{3}\right)=1$, which in turn implies that

$$
R_{2}\left(N, p, k, \beta_{1}, \beta_{2}, \beta_{3}\right) \geq R_{2}\left(N, p, N, \beta_{1}, \beta_{2}, \beta_{3}\right)=1,
$$

and this completes the proof.

As an application of Theorem 2, suppose that $\beta_{1}=\beta_{3}=1, \beta_{2}=0.9, N=1000, a=5$, and $p=10^{-5}$. Then, from (7) and (8), $\mathrm{E}\left(Y_{2}\right) \approx 0.1$ and $\operatorname{var}\left(Y_{2}\right) \approx 50$. Now, from Theorem 2 ,

$$
R_{1}\left(N, p, \beta_{1}, \beta_{2}, \beta_{3}\right) \approx \exp \{-0.01\}[\exp \{-0.01+0.25\}]^{-1}=\exp \{-0.25\}=0.78 .
$$

This means that a Type I nanocomponent with this specification has a reliability of 0.78 . Here a Type II nanocomponent has a reliability of 1 . 


\subsection{Bounds for reliabilities}

It is clear that in general it is possible to assess the nanocomponent reliability provided that we can specify all the parameters in (4). However, such information may sometimes be unavailable or difficult to obtain. Thus, in many practical situations it is desirable or necessary to check whether or not the reliability of the nanocomponent meets a given specification when some of the parameters are known. If a lower bound in the foregoing already meets or exceeds the reliability, then we know for sure that the system meets the specification. As mentioned, such conclusions are gratifying, particularly when evaluations of $R_{1}\left(N, p, \beta_{1}, \beta_{2}, \beta_{3}\right)$ and $R_{2}\left(N, p, k, \beta_{1}, \beta_{2}, \beta_{3}\right)$ are not feasible.

The following theorem gives bounds for $R_{1}\left(N, p, k, \beta_{1}, \beta_{2}, \beta_{3}\right)$ and $R_{2}\left(N, p, \beta_{1}, \beta_{2}, \beta_{3}\right)$.

Theorem 3. If $\beta_{1} \geq \beta_{2}$ and $\beta_{3} \geq \beta_{2}$, then

(a) $R_{1}\left(N, p, \beta_{1}, \beta_{2}, \beta_{3}\right) \geq q^{N}$,

(b) $R_{2}\left(N, p, k, \beta_{1}, \beta_{2}, \beta_{3}, k\right) \geq \max _{\Omega(k, N)} \prod_{j=1}^{k}\left(1-\frac{p^{2} \exp \left\{\left(\beta_{1}-\beta_{2}\right) a\left(i_{j}\right)\right\}}{q+p \exp \left\{\left(\beta_{1}-\beta_{2}\right) a\left(i_{j}\right)\right\}}\right)$,

where $a\left(i_{j}\right)$ is the number of atoms neighboring the atom located at $s\left(i_{j}\right)$.

Proof. Part (a) follows from Lemma 1. To prove part (b), following Barlow and Proschan (1981, pp. 29-31), from (11) and the fact that $Y_{1}^{*}, \ldots, Y_{N}^{*}$ are associated, we obtain

$$
R_{2}\left(N, p, k, \beta_{1}, \beta_{2}, \beta_{3}\right) \geq \max _{\Omega(k, N)} \mathrm{P}\left(Y_{i_{1}}^{*}=0, \ldots, Y_{i_{k}}^{*}=0\right) \geq \max _{\Omega(k, N)} \prod_{j=1}^{k} \mathrm{P}\left(Y_{i_{j}}^{*}=0\right) .
$$

Now, for any $i$,

$$
\begin{aligned}
\mathrm{P}\left(Y_{i}^{*}=0\right) & =1-\mathrm{P}\left(Y_{i}^{*}=1\right) \\
& =1-\mathrm{P}\left(X\left(s_{i}\right) \prod_{j \in N^{*}\left(s_{i}\right)} X\left(s_{j}\right)=1\right) \\
& =1-\mathrm{P}\left(X\left(s_{i}\right)=1 \mid \prod_{j \in N^{*}\left(s_{i}\right)} X\left(s_{j}\right)=1\right) \mathrm{P}\left(\prod_{j \in N^{*}\left(s_{i}\right)} X\left(s_{j}\right)=1\right) \\
& =1-\frac{p \exp \left\{\left(\beta_{1}-\beta_{2}\right) a(i)\right\}}{q+p \exp \left\{\left(\beta_{1}-\beta_{2}\right) a(i)\right\}} \mathrm{P}\left(X\left(s_{j}\right)=1, j \in N^{*}\left(s_{i}\right)\right) \\
& \geq 1-\frac{p^{2} \exp \left\{\left(\beta_{1}-\beta_{2}\right) a(i)\right\}}{q+p \exp \left\{\left(\beta_{1}-\beta_{2}\right) a(i)\right\}} .
\end{aligned}
$$

The result follows by replacing $\mathrm{P}\left(Y_{i_{j}}^{*}=0\right)$ in (12) with (13). Note that the third equality in (13) comes from (5).

In Theorem 3, if $a(i)=a, i=1, \ldots, N$, then the second part of Theorem 3 reduces to

$$
R_{2}\left(N, p, k, \beta_{1}, \beta_{2}, \beta_{3}\right) \geq\left(1-\frac{p^{2} \exp \left\{\left(\beta_{1}-\beta_{2}\right) a\right\}}{q+p \exp \left\{\left(\beta_{1}-\beta_{2}\right) a\right\}}\right)^{k} .
$$

For example, if $\beta_{1}=2, \beta_{2}=1, p=0.1, a=2$, and $k=5$, then

$$
R_{2}\left(N, p, k, \beta_{1}, \beta_{2}, \beta_{3}\right) \geq\left(1-\frac{(0.01) \mathrm{e}^{2}}{0.9+0.1 \mathrm{e}^{2}}\right)^{5} \approx 0.8 \text {. }
$$


This means that, for a Type II nanocomponent with this specification, the reliability is at least 0.80 .

\subsection{Assessing reliability for the nonsparse case}

In this section we use the Gibbs sampler to approximate the reliabilities of Type I and Type II nanocomponents. See Casella and George (1992) for more details about the Gibbs sampler. For this, we first generate observations from the joint distribution of $X\left(s_{1}\right), \ldots, X\left(s_{N}\right)$ given in (4). The algorithm and how to implement it are described below.

(a) Randomly set the value of $X\left(s_{i}\right)$ with $\mathrm{P}\left(X\left(s_{i}\right)=1\right)=p, i=1, \ldots, N$.

(b) For a given atom, say atom $i$ located at $s_{i}$, update the value of $X\left(s_{i}\right)$ using (5).

(c) Repeat step (b) a specific number of times until all conditional probabilities are stabilized.

Now, to approximate $R_{1}\left(N, p, \beta_{1}, \beta_{2}, \beta_{3}\right)$, repeat steps (a)-(c) several times, say $n$ times, to generate samples of size $M$ from $X\left(s_{1}\right), \ldots, X\left(s_{N}\right)$. Now, if $M_{1}$ is the number of times that $X\left(s_{1}\right)=\cdots=X\left(s_{N}\right)=0$ then the approximate reliability of a Type I nanocomponent is $M_{1} / M$.

Now to approximate the reliability of a Type II nanocomponent, suppose that $M_{2}$ is the number of times that $W_{N}$ is greater than or equal to $k$. Then, $R_{2}\left(N, p, k, \beta_{1}, \beta_{2}, \beta_{3}\right)$ can be approximated by $M_{2} / M$.

Our proposed algorithm was implemented using the $\mathrm{R}$ program. A description of this algorithm, which is referred to as 'nano', is given next.

Algorithm 1. (nano(N, p, beta1, beta2, beta3, neighbor, $k$, burnin, lag, $n)$.)

Description. A function to compute the reliabilities of Type I and Type II nanocomponents.

Parameters. N: the number of atoms (an integer).

p: probability $p$ in (5) (a numeric).

beta1: parameter $\beta_{1}$ in (5) (a numeric).

beta2: parameter $\beta_{2}$ in (5) (a numeric).

beta3: parameter $\beta_{3}$ in (5) (a numeric).

neighbor: the neighbors of each atom (a list of length $N$ ).

$\mathrm{k}$ : $k$ in the computation of the Type II reliabilities (an integer).

burnin: burn-in period (an integer).

lag: lag period (an integer).

$\mathrm{n}$ : the number of iterations (an integer).

Return. OBS: the sample matrix with each row denoting one sample.

type1: the reliability for a Type I nanocomponent.

type2: the reliability for a Type II nanocomponent.

\section{Subroutine.}

1. Randomly set the values of $X\left(s_{i}\right)$ with $\mathrm{P}\left(X\left(s_{i}\right)=1\right)=p, i=1, \ldots, N$.

2. For a given atom, say atom $i$ located at $s_{i}$, update the value of $X\left(s_{i}\right)$ using (5).

3. Repeat step $2 n$ times. 
4. Select samples using the burn-in and lag periods, getting samples of size

$$
M=\text { integer }\left(\frac{n-\text { burnin }-1}{\operatorname{lag}}\right)+1 .
$$

(a) Compute $M_{1}$, the number of times that $X\left(s_{1}\right)=\cdots=X\left(s_{N}\right)=0$; type 1 is then $M_{1} / M$.

(b) In each sample, for each atom $i$, compute $Y_{i}$, the product of the status of atom $i$ and the status of its neghbors, and further compute $W_{N}=N-\sum_{1}^{N}\left(Y_{i}\right)$.

(c) Compute $M_{2}$, the number of times that $W_{N} \geq k$; type2 is then $M_{2} / M$.

Code.

nano $\leftarrow$ function(N,p, beta1, beta2, beta3, neighbor,k, burnin,lag,n)

\{

if(N!=length(neighbor)) cat("neighbor structure is wrong...")

else

\{

$\mathrm{cp} \leftarrow$ function(prob,b1,b2,b3,11,10)

$\{\mathrm{x} \leftarrow$ prob*exp $((\mathrm{b} 1-\mathrm{b} 2) * 11+(\mathrm{b} 2-\mathrm{b} 3) * 10) ; \mathrm{y} \leftarrow \mathrm{x}+1-$ prob; $\mathrm{x} / \mathrm{y}\}$

$\mathrm{L} \leftarrow \operatorname{rbinom}(\mathrm{N}, 1, \mathrm{p}) ; \mathrm{CP} \leftarrow \mathrm{rep}(\mathrm{p}, \mathrm{N}) ; \mathrm{M} \leftarrow \operatorname{matrix}(\mathrm{ncol}=\mathrm{N}$, nrow $=\mathrm{n})$;

for $(\mathrm{j}$ in $1: \mathrm{n})\{$ for $(\mathrm{i}$ in $1: \mathrm{N})\{\mathrm{L} 1 \leftarrow \operatorname{sum}(\mathrm{L}[$ neighbor[[i]]]); L0 $\leftarrow$ length(neighbor[[i]])-L1;

$\mathrm{CP}[\mathrm{i}] \leftarrow \mathrm{cp}($ p, beta1, beta2, beta3,L1,L0); $\mathrm{L}[\mathrm{i}] \leftarrow \operatorname{rbinom}(1,1, \mathrm{CP}[\mathrm{i}])\} \mathrm{M}[\mathrm{j},] \leftarrow \mathrm{L}\}$

OBS $\leftarrow \mathrm{M}[\mathrm{c}($ burnin +1 , burnin+1+(1:as.integer $(($ n-burnin-1)/lag $)) * \operatorname{lag})$,$] ;$

row.sums $\leftarrow$ apply(OBS, 1 ,sum); $\mathrm{WN} \leftarrow \operatorname{vector}() ;$

for(i in 1:nrow(OBS) $\{\mathrm{V} \leftarrow \operatorname{vector}()$;

for $(\mathrm{j}$ in $1: \mathrm{N}) \mathrm{V}[\mathrm{j}] \leftarrow \mathrm{OBS}[\mathrm{i}, \mathrm{j}] * \operatorname{prod}(\mathrm{OBS}[\mathrm{i}$, neighbor$[[\mathrm{j}]]])$;

$\mathrm{WN}[\mathrm{i}] \leftarrow \mathrm{N}-\operatorname{sum}(\mathrm{V})\}$

return(list $(\mathrm{OBS}=\mathrm{OBS}$,type $1=$ length(which(row.sums==0))/length(row.sums), type2=length(which $(\mathrm{WN}>=\mathrm{k})) /$ length $(\mathrm{WN}))$ )

\}\}

As an application, we give the following example.

Example 1. Consider a nanocomponent consisting of six atoms, i.e. $N=6$. For atoms $1,2, \ldots, 6$, suppose that their corresponding neighbors are $\{2,3\},\{1,3,4\},\{4,5,6\},\{3,4,5\}$, $\{1,2,3,4\}$, and $\{1,5\}$, respectively. Also, we let $p=0.8, \beta_{1}=2, \beta_{2}=1, \beta_{3}=3, k=3$, burnin $=100$, lag $=10$, and $n=5000$. Run 'nano' as nano(6, 0.8, 2, 1, 3, neighbor, 3, 100, 10, 5000). The results are: type $1=0.4$ (Type I nanocomponent reliability), type $2=0.5693878$ (Type II nanocomponent reliability). Here, $M=490$. We can also compute the $(1-\alpha) \%$ confidence intervals for the reliabilities of Type I and Type II structures using the equations

$$
\frac{M_{1}}{M} \pm z_{\alpha / 2} \sqrt{\frac{M_{1}\left(1-M_{1} / M\right) / M}{M}} \text { and } \frac{M_{2}}{M} \pm z_{\alpha / 2} \sqrt{\frac{M_{2}\left(1-M_{2} / M\right) / M}{M}}
$$

respectively. In this example, the $95 \%$ confidence intervals are $(0.38,0.42)$ and $(0.55,0.59)$ for the reliabilities of Type I and Type II, respectively.

\section{Conclusions}

In this paper we have proposed a model to capture interactions between atoms of a nanocomponent which is an extension of the model in Ebrahimi (1998). We studied several properties 
of this model. We then used these properties to assess reliabilities of nanocomponents with two different structures for a known mission time or the present moment $t_{0}$.

\section{Acknowledgements}

The authors wish to thank the anonymous referee for some important suggestions about improvements both in the form and in the content. Nader Ebrahimi's research was partially supported by the National Security Agency, under grant number H98230-11-1-0138. The United States Government is authorized to reproduce and distribute reprints notwithstanding any copyright notion herein.

\section{References}

Barlow, R. E. And Proschan, F. (1981). Statistical Theory of Reliability and Life Testing. To Begin With, Silver Spring, MD.

BesAG, J. (1974). Spatial interaction and the statistical analysis of lattice systems. J. R. Statist. Soc. B 36, $192-236$.

Bloemena, A. R. (1964). Sampling from a Graph (Math. Centre Tracts 2). Mathematisch Centrum, Amesterdam.

Casella, G. and George, E. I. (1992). Explaining the Gibbs sampler. Amer. Statistician 46, 167-174.

Deng, M., Chen, T. And Sun, F. (2004). An integrated probabilistic model for functional prediction of proteins. J. Comput. Biol. 11, 463-475.

Ebrahimi, N. (2008). Assessing a linear nanosystem's limiting reliability from its components. J. Appl. Prob. 45, 879-887.

EBRAHimi, N. (2010). Assessing 2-dimensional nanocomponent's limiting reliability. IEEE Trans. Reliab. 59, $139-144$.

SAUnders, R., KRYSCIO, R. AND FunK, G. M. (1979). Limiting results for arrays of binary random variables on rectangular lattices under sparseness conditions. J. Appl. Prob. 16, 554-566. 